\title{
Delivery of isoniazid preventive therapy to reduce occupational TB among healthcare workers in Swaziland
}

\author{
aUniversity Research Co., LLC, Swaziland \\ bSwaziland National AIDS Program, Swaziland \\ 'USAID/PEPFAR, Swaziland \\ ${ }^{d} C D C$, Swaziland \\ eUniversity Research Co., LLC, Bethesda, MD, USA \\ *Corresponding author, email: vghazaryan@urc-chs.com
}

Marianne Calnan ${ }^{a}$, Samson Haumba ${ }^{a}$, Makhosazana Matsebula ${ }^{a}$, Ntombifuthi Shongwe ${ }^{a}$, Munyaradzi Pasipamire ${ }^{b}$, Natalie Kruse Levyc, Munamato Mirirac, Peter Preko d, Alisha Smith-Arthure and Varduhi Ghazaryan ${ }^{\mathrm{e} *}$

Healthcare workers (HCWs) in Swaziland are at increased risk of acquiring tuberculosis (TB), but existing infection control and occupational health policies often fall short of mitigating the risk of acquiring TB in the workplace. Health service failure to systematically offer isoniazid preventive therapy (IPT) to HCWs, which is endorsed by the World Health Organisation (WHO), contributes significantly to the TB incidence among HCWs in high HIV prevalence settings. This paper describes a pilot introduction of IPT to HCWs, and expounds possible determinants for adherence and non-acceptance to IPT. Acceptance to the widespread use of IPT among HCWs can be improved through education on risk and counselling.

Keywords: healthcare workers, infection control, isoniazid preventive therapy, occupational health and safety, tuberculosis

\begin{abstract}
Introduction
The increased risk of tuberculosis (TB) infection among healthcare workers (HCWs) is well established and widespread, encompassing not just direct providers but also a range of health personnel and auxiliary staff in healthcare settings, particularly in high TB burden countries. ${ }^{1-4}$ This can lead to absenteeism due to illness or death, and the perceived risk of acquiring TB may negatively impact a health provider's performance. ${ }^{5}$
\end{abstract}

Occupational health and safety strategies to protect HCWs in settings with a high TB burden have lagged, and tend to focus on strengthening traditional infection control measures, including improving administrative and environmental controls and increasing the use of personnel protective equipment. The consistent application of these measures is often hampered by health system constraints, including weak infrastructure, lack of resources for infection control materials, and poor provider knowledge and awareness. ${ }^{4,6}$ A number of factors, including job roles, duration of employment, and availability of personal protective equipment may impede a worker's ability to reduce the risk of exposure to TB over the long term. ${ }^{3}$

The administration of prophylactic therapy for HCWs at high TB burden settings is a potential strategy for reducing TB incidence. Prophylactic therapy in the form of isoniazid-based preventive therapy (IPT) is a commonly adopted approach for reducing the risk of developing active TB disease among high-risk groups, such as people living with the human immunodeficiency virus (PLHIV) and children under 5 who are exposed to TB. The World Health Organisation (WHO) endorses a strategy for preventing TB among these high-risk groups based on administration of isoniazid for six months, and calls for a case-by-case evaluation and further operational research regarding the provision of IPT among HCWs.? The routine provision of IPT to HCWs, requires that adequate systems are in place to establish HCWs' HIV status, rule out active TB disease, and monitor latent TB infection (LTBI). To date, the operational and ethical challenges have prevented many healthcare settings from attempting IPT provision for $\mathrm{HCWs}$, though some evidence suggests that the introduction of IPT reduced the relative lifetime risk of active TB for HCWs. ${ }^{1} \mathrm{~A}$ literature review of evidence from tuberculin skin testing and chemoprophylaxis programs among HCWs conducted in 2011 yielded only three examples from high TB prevalence settings, while supporting a recommendation that IPT should be provided to HCWs with LTBI once active TB is ruled out. ${ }^{8}$ The operational dimensions of this recommendation remain to be explored in many countries.

\section{Aim and objectives}

The overall aim of this paper is to describe the experience of introducing prophylactic TB treatment to HCWs in Swaziland. The objectives are to conduct quantitative analysis on IPT provision data at 12 wellness pilot sites, and to conduct qualitative analysis on HCW experience with IPT. Swaziland has the highest estimated incidence of TB in the world. ${ }^{9}$ The country also has the highest prevalence of HIV in the world. ${ }^{10}$ We are not aware of any specific studies of TB disease among HCWs in Swaziland, but studies in South Africa have demonstrated that the TB burden among HCWs is three times as high as in the general population, and that incidence of LTBI may be twice as high among HCWs as the general population. ${ }^{11,12}$ The risk of occupational exposure to TB in health settings in Swaziland is acute, and fear of TB has resulted in organised action by HCWs in the form of labour strikes.

\section{Process of introducing IPT for HCWs}

In 2009, IPT was introduced by the national TB program and technical assistance partners in 12 sites in Swaziland on a pilot basis. Eligibility for IPT focused on pre-antiretroviral therapy (ART) and ART patients. Following the pilot, IPT was introduced 
Table 1: Profile of HCW respondents, disaggregated by gender

\begin{tabular}{|c|c|c|}
\hline Age & Female $(n=13)$ & Male $(n=6)$ \\
\hline $25-34$ years & 3 & 3 \\
\hline $35-44$ years & 5 & 2 \\
\hline $44+$ years & 5 & 1 \\
\hline Total & 13 & 6 \\
\hline \multicolumn{3}{|l|}{ Job classification } \\
\hline Registered nurse & 3 & \\
\hline Nursing assistant & 2 & 1 \\
\hline Staff nurse & 2 & 1 \\
\hline Case worker & 1 & \\
\hline Nursing student & 1 & \\
\hline
\end{tabular}

*One respondent did not provide her job classification.

nationwide for all PLHIV without active TB. Expansion of the IPT program was accomplished through consecutive national trainings starting in 2011. In December 2012, focus group discussions were held by University Research Company, LLC (URC) in collaboration with the Swaziland National AIDS Program targeting HIV and TB clinic staff, community health workers, peer supporters, current patients attending the HIV and TB clinics, and community members from southern Hhohho region, followed by a progress review of the IPT program in May 2013. These investigations identified recurrent impediments to implementation, including the inconsistent availability of isoniazid, the lack of standardised recording and reporting tools for IPT, and conflicting views on the importance of IPT, including perceptions of increased pill burden and increased clinic visits. Additional barriers included weaknesses in communication between the facilities and central medical stores, and frequent turnover of personnel who had been trained to administer IPT.

Although the national IPT policy focuses on provision for people who are infected with HIV, the national TB management guidelines emphasise provision of IPT to HCWs. However, the introduction of IPT to HCWs was not prioritized until a national pilot program of TB screening among HCWs was initiated in early 2012 in the wellness clinics that cater to HCWs. IPT based on a six-month course of treatment was first offered to HCWs in June 2012. All HCWs visiting the wellness clinics were screened for TB symptoms and eligibility for IPT. A HCW was deemed ineligible for IPT if they were suffering from liver disease, had symptoms or signs of TB, recent history of contact with multidrug resistant TB, previous history of sensitivity to isoniazid, alcohol dependence, or had taken isoniazid in the preceding two years. Tuberculin skin tests are not routinely offered in Swaziland, and HCWs did not receive one prior to IPT.

\section{Methods}

Between February and April 2015, quantitative data were collected from facility IPT log books on numbers of HCWs who had been screened for TB, offered IPT, and started and completed IPT between 2012 and 2014; and 19 qualitative interviews collected data on experiences and attitudes towards IPT from a sample of HCWs attending 16 health worker occupational clinics, which actively engaged in providing TB screening among HCWs.

\section{Results}

Between 2013 and 2014, a total of 4878 HCWs were screened for TB, and 3268 (67\%) were reported eligible for IPT. However, only 687 (21\%) were started on IPT. From 2012-2014, 914 HCWs were started on IPT nationwide but there were no records of TB screening for the 2012 cohort. Of these, only 500 are reported as having completed the six-month treatment course. Reflecting weaknesses in reporting, $260 \mathrm{HCWs}$ who started treatment in 2012 (143) and 2013 (117) are listed as currently on IPT. Reporting of IPT data improved in 2014, perhaps reflecting improvements in service delivery systems. In 2014, $176 \mathrm{HCW}$ sere started on IPT and 154 completed, six discontinued, three were lost to follow up, and ten were still on IPT at the time of this review. Only three outcomes are not accounted for in 2014, which can be considered an achievement of the reporting system. From 20122014 , only 14 HCWs $(<2 \%)$ who started on IPT reported side effects, which is significant as HCWs may have chosen not to take IPT due to concern about potential side effects, though it is unclear whether instances of side effects may have gone unreported or were not adequately captured in the recording tools.

Between $14^{\text {th }}$ and $16^{\text {th }}$ April 2014, a total of 19 qualitative interviews with HCWs at the $16 \mathrm{HCW}$ wellness areas were conducted; these are profiled in Table 1.

Among the participants in the qualitative interviews, 10\% (2) respondents had previously had TB, 47\% (9) had been offered IPT and $53 \%$ (10) had not been offered IPT. Among HCWs with TB, expressions of frustration on barriers they faced in getting information about their risk are common. Implementation of a screening and prophylaxis program may have the effect of boosting morale and reducing attrition. Among those who had been offered IPT, 33\% (3) reported that they had accepted; of these, $67 \%$ (2) had completed the full course, and one was still on treatment.

Among those who had chosen to take IPT, the motivation was a desire to reduce their risk of getting TB. None of the HCWs who had taken IPT reported experiencing any side effects, nor did they report challenges in accessing the full course of medication.

For those who had been offered IPT but had not accepted, the reasons for non-acceptance included confusion about eligibility, 
skepticism that IPT reduces the risk of getting TB, fear of longterm isoniazid resistance, and concern over availability of the medication. However, among those who had never been offered IPT, non-acceptance would be influenced by concerns about side effects, and several HCWs in this group demonstrated poor awareness or understanding of IPT, including eligibility and how to access it.

Participants in general had a positive attitude towards IPT but stressed that the strongest determinants on acceptance of IPT was better information and uninterrupted availability of IPT. The need to counter misinformation about IPT was the other recurrent theme. To this end, several participants recommended that IPT should be offered to all HCWs, and that more campaigns should be conducted to reach HCWs with IPT, in conjunction with efforts to improve systematic TB screening for HCWs. They recommended also the need for better sensitisation and education to motivate HCWs to take IPT especially emphasising the importance of IPT in reducing TB risk. They also recommended the need to counter misinformation about IPT. In addition, participants suggested that acceptance and adherence could be improved by use of counselling and IPT support groups. The responses, overall, indicated a high level of awareness of the need for HCWs to have the means to reduce their TB risk.

\section{Discussion}

Failure of the health service to provide IPT and IPT information are major barriers in the implementation of WHO guidance on IPT for HCWs. Availability of IPT for HCWs has been improving in Swaziland since 2012, but avoided by several barriers including systematic documentation of IPT provision and outcomes. Although the review of the IPT provision data shows that fewer HCWs received IPT in 2014 than in previous years, a significantly higher proportion of HCWs were documented to have completed their course of IPT, indicating improvements in service delivery, follow-up, and recording systems. The qualitative interview results, although capturing a limited sample of HCWs, revealed the key themes emerging are acceptability, non-acceptability, adherence, support systems and IPT information, demonstrating that HCWs are interested in receiving IPT if the delivery systems are in place to improve access and support adherence. Persistent service delivery challenges have been experienced in ensuring the availability of isoniazid, and there is a need for strengthening reporting and follow-up of HCWs once started on IPT. Data recording and reporting tools are needed that capture treatment outcomes progressively so that HCWs on IPT can be followed up and their status reflected until completion, accompanied by a stronger emphasis on routine data recording. Responsibility for IPT provision needs to be shared across clinic staff to ensure that personnel are always available for service provision.

In order to improve acceptability of IPT for HCWs, a patientfriendly, service-oriented environment is needed with efforts to increase education around how IPT can protect HCWs by reducing their risk of $\mathrm{TB}$, including practical information about how to access IPT. Information channels and service delivery systems need to be oriented and tailored towards HCWs to minimise the obstacles they experience and promote a sense that their needs are being met. Better information and stronger counselling are also needed to counter fears of stigma associated with IPT, as HCWs continue to associate IPT with a positive HIV status. The current experience from the pilot study demonstrates that the addition of healthcare worker wellness areas in clinics is an effective way of improving provision of TB screening and IPT.

\section{Conclusion}

Provision of IPT for HCWs at high risk of TB requires expanding existing as well as establishing new service delivery models, including use of occupational wellness sites. This review of the pilot implementation shows that HCWs want more proactive and supportive systems in place to protect them from TB. The need for better sensitisation and education to motivate HCWs to take IPT was a recurring theme, including emphasising the importance of IPT to reduce TB risk and to counter misinformation. Further studies with larger sample sizes to better understand determinants of IPT acceptance and adherence among HCWs as well as service delivery models and support systems are recommended.

Acknowledgement - We thank our colleagues and staff at the Wellness Center Manzini and facilities that participated in the Health Care Worker TB screening pilot. Appreciation is extended to the HCWs who assisted to gather data and participated in the survey.

Declaration - The authors gratefully acknowledge the United States Agency for International Development (USAID) and the U.S. President's Emergency Plan for AIDS Relief (PEPFAR) for funding the USAID Health Care Improvement Project and the USAID Applying Science to Strengthen and Improve Systems Project in Swaziland, under which the interventions described in the paper were implemented. The USAID ASSIST Project is managed by University Research Co., LLC under Cooperative Agreement Number AID-OAA-A-12-00101. The USAID Health Care Improvement Project was managed by URC under Contract Number GHN-I-03-07-00003-00.

Conflict of interest - The authors do not have a commercial or other association that may have posed a conflict of interest.

\section{References}

1. Raj $\mathrm{R}$, Prasad $\mathrm{H}$, Arya BK, et al. Isoniozid preventive therapy programmes for healthcare workers in India: translating evidence into policy. Natl Med J India. 2011;24(4):201-7.

2. Kassim S, Zuber P, Wiktor SZ, et al. Tuberculin skin testing to assess the occupational risk of Mycobacterium tuberculosis infection among health care workers in Abidjan, Côte d'Ivoire. Int J Tuberc Lung Dis. 2000;4(4):321-6.

3. Sherman HA, Karakis I, Heimer D, et al. Housekeeping health care workers have the highest risk for tuberculin skin test conversion. Int Tuberc Lung Dis. 2011;15(8):1050-5.

4. Tudor C, Van der Walt M, Hill MN, et al. Occupational health policies and practices related to tuberculosis in health care workers in KwaZulu-Natal, South Africa. Public Health Action. 2013;3(2):141-145.

5. Padayatchi N, Daftary A, Moodley T, et al. Case series of the long-term psychosocial impact of drug-resistant tuberculosis in HIV-negative medical doctors. Int J Tuberc Lung Dis. 2010;14(8):960-6.

6. Brouwer M, Coelho E, Mosse CD, et al. Healthcare workers' challenges in the implementation of tuberculosis infection prevention and control measures in Mozambique. PLoS ONE. 2014;9(12):e114364.

7. World Health Organization. WHO policy on TB infection control in health-care facilities, congregate settings and households; 2009. Available from: http://www.who.int/tb/publications/2009/infection_ control/en/.

8. Khawcharoenporn T, Apisarnthanarak A, Sungkanuparph $\mathrm{S}$, et al. Tuberculin skin test and isoniazid prophylaxis among health care workers in high tuberculosis prevalence areas. Int J Tuberc Lung Dis. 2011;15(1):14-23.

9. World Health Organization. Global tuberculosis control report; 2014. WHO/HTM/TB2014. Available from: http://www.who.int/tb/ publications/global_report/en/ 
10. Bicego G, Nkambule R, Perterson I, et al. Recent Patterns in Population-Based HIV Prevalence in Swaziland. PLoS ONE. 2013. doi:10.1371/journal.pone.0077101

11. Claassens MM, van Schalkwyk C, du Toit E, et al. Tuberculosis in healthcare workers and infection control measures at primary healthcare facilities in South Africa. PLoS ONE 2013;8(10):e.76272
12. McCarthy KM, Scott LE, Gous $N$, et al. High incidence of latent tuberculous infection among South African health workers: an urgent call for action. Int J Tuberc Lung Dis. 2015;19(6):647-53.

Received: 05-10-2015 Accepted: 19-04-2016 